\title{
Global dynamics for a class of non-monotone time-delayed reaction-diffusion equations
}

\author{
Yueding Yuan ${ }^{1,2^{*}}$ (D) and Haibo Chen
}

\section{"Correspondence:}

yuanyueding2017@163.com

${ }^{1}$ School of Mathematics and

Statistics, Central South University,

Changsha, P.R. China

${ }^{2}$ School of Mathematics and

Computer Sciences, Yichun

University, Yichun, P.R. China

\section{每 Springer}

\begin{abstract}
This paper deals with a large class of non-monotone time-delayed reaction-diffusion equations in which the reaction term can be spatially nonlocal. Nonexistence, existence, uniqueness and global attractivity of positive equilibriums to the equation are addressed. In particular, developed is a technique that combines the method of super-sub solutions, the variation-of-constants formula for the delay differential equation and the estimation of integral kernels, which enables us to obtain some sufficient conditions for the global attractiveness of the unique positive equilibrium. Two examples are given to illustrate the obtained results.
\end{abstract}

Keywords: global attractivity; delay equations; positive equilibriums; existence and uniqueness

\section{Introduction}

Consider the following non-monotone reaction-diffusion equation with time delay:

$$
\left\{\begin{array}{l}
\frac{\partial u(t, x)}{\partial t}=d \Delta u(t, x)-g(u(t, x))+\mu \int_{\bar{\Omega}} \rho(\eta, x, y) f(u(t-\tau, y)) d y, \quad t>0, x \in \Omega, \\
B u(t, x)=0, \quad t>0, x \in \partial \Omega .
\end{array}\right.
$$

Here, $d>0, \mu>0, \eta \geq 0, \tau>0 . \Delta$ is the Laplacian operator on $\mathbb{R}^{m}$, where $m \geq 1$ and $\mathbb{R}=(-\infty,+\infty) . \Omega$ is a bounded, open and convex domain with a smooth boundary $\partial \Omega$ in $\mathbb{R}^{m} . \bar{\Omega}$ is the closure of $\Omega . x \in \Omega$ denotes $x$ belonging to $\Omega$. Either $B u=u$ or $B u=\partial u / \partial \mathbf{n}$, where $\partial / \partial \mathbf{n}$ denotes the differentiation in the direction of the outward normal $\mathbf{n}$ to $\partial \Omega$. And the kernel function $\rho(\eta, x, y)$ is the fundamental solution associated with the operator $\partial_{\eta}-\Delta_{x}$ and boundary condition $B u=0$. Such an equation arises from the interaction of intrinsic dynamics and the spatial diffusion in a structured population [1]. When $u$ in (1.1) is regarded as the density of the mature population in a two-stage population (mature and immature, with a fixed maturation time $\tau$ ), then $g(u)$ and $f(u)$ are the death function and the birth function of mature individuals, respectively, and the term $\int_{\bar{\Omega}} \rho(\eta, x, y) f(u(t-$ $\tau, y)) d y$ is exactly the maturation rate of those immature individuals born at time $\tau$ ago. For the model derivation and historical accounts of the development, see Gourley and Wu's survey article [2] and the references therein.

(c) The Author(s) 2018. This article is distributed under the terms of the Creative Commons Attribution 4.0 International License (http://creativecommons.org/licenses/by/4.0/), which permits unrestricted use, distribution, and reproduction in any medium, provided you give appropriate credit to the original author(s) and the source, provide a link to the Creative Commons license, and indicate if changes were made. 
To make clear of the global dynamics to (1.1), a central question is to investigate existence, uniqueness and global attractivity of positive equilibriums. If the birth function $f(u)$ always increases with $u>0$, the monotone dynamical system approach can be applied, and this central question has been solved very well [3]. However, in the real world, the birth function $f(u)$ is often non-monotone. It has been more common to assume that $f(u)$ decreases for sufficiently large $u$. For example, researchers often assume that $f(u)=p u(q-u)$ in the most prevailing logistic model, $f(u)=\frac{p u}{q+u^{\beta}}$ in the Mackey-Glass model and $f(u)=p u e^{-q u}$ in the Nicholson's blowflies model, where $p, q, \beta>0$. For nonmonotone $f(u)$, the theory of monotone dynamical systems may not be adequate, and this question becomes very subtle. This motivates many authors to investigate this central question for some special cases of (1.1) [4-11]. For example, Zhao [11] established the global attractivity of the positive constant steady state for the special case of $B u=\partial u / \partial \mathbf{n}$ and $g(u)=\alpha u$ of (1.1) by using a fluctuation method [12], where $\alpha>0$. Guo et al. [4] studied the special case of $B u=u, \Omega=(0, \pi), g(u)=\alpha u$ and $\eta>0$. By employing the method of super-sub solution, combined with the careful analysis for $\rho(\eta, x, y)$, they obtained some sufficient conditions for nonexistence, existence and uniqueness of positive equilibriums [4]. At the same time, Yi and Zou [7] analyzed the special case of $B u=u, g(u)=\alpha u$ and $\eta>0$. By using the comparison technique and the theory of monotone dynamical systems, they found some sufficient conditions for the existence and global attractiveness of positive equilibriums [7]. For the general case of (1.1), this central question remained unsettled.

These observations motivate us to study the global asymptotic behavior for (1.1). For convenience, we consider the following version:

$$
\left\{\begin{array}{l}
\frac{\partial u(t, x)}{\partial t}=d \Delta u(t, x)-g(u(t, x))+\int_{\bar{\Omega}} \rho(\eta, x, y) f(u(t-\tau, y)) d y, \quad t>0, x \in \Omega, \\
B u(t, x)=0, \quad t>0, x \in \partial \Omega, \\
u(\theta, x)=\varphi(\theta, x), \quad \theta \in[-\tau, 0], x \in \Omega
\end{array}\right.
$$

where $\varphi:[-\tau, 0] \times \Omega \rightarrow \mathbb{R}$ is a bounded, continuous and positive initial function. By the derivation in [3], the kernel function $\rho(\eta, x, y)$ is given by

$$
\rho(\eta, x, y)= \begin{cases}\sum_{n=1}^{+\infty} e^{-\eta \xi_{n}} \psi_{n}(x) \psi_{n}(y), & \text { if } \eta>0, \\ \delta(x-y), & \text { if } \eta=0 .\end{cases}
$$

Here, $0 \leq \xi_{1}<\xi_{2} \leq \cdots \leq \xi_{n} \leq \cdots$ with $\lim _{n \rightarrow \infty} \xi_{n}=+\infty$ are the eigenvalues of the operator $-\Delta$ subject to the homogeneous Neumann (or Dirichlet) boundary condition on $\partial \Omega$. The function $\psi_{n}$ is the eigenvector corresponding to $\xi_{n} .\left\{\psi_{n}\right\}_{n=1}^{+\infty}$ is a complete orthonormal system in the Banach space $L^{2}(\bar{\Omega}) . \psi_{1}(x)>0$ for all $x \in \Omega$. And $\delta(x)$ is the Dirac function on $\mathbb{R}^{m}[11,13]$. For any function $a(x)$, we always denote by $a^{\prime}(x)$ the derivative with respect to $x$ of the function $a(x)$. Throughout this paper, we assume that:

(A1) $f \in C^{1}\left(\mathbb{R}^{+}, \mathbb{R}^{+}\right), f(u)=u h_{1}(u), h_{1}(u)>0$ and $h_{1}^{\prime}(u)<0$ for all $u \geq 0$, where $\mathbb{R}^{+}=[0,+\infty)$.

(A2) Both $f(u)$ and $f^{\prime}(u)$ are bounded for $u \geq 0$.

(A3) $g \in C^{1}\left(\mathbb{R}^{+}, \mathbb{R}^{+}\right), g(u)=u h_{2}(u), h_{2}(u)>0$ and $h_{2}^{\prime}(u) \geq 0$ for all $u \geq 0$.

(A4) There exists a real number $M>0$ such that $\hat{f}(u)<g(u)$ for all $u>M$, where $\hat{f}(u)=\max _{v \in[0, u]} f(v)$. 
(A5) There exists a positive real number $M_{0}$ such that $h_{2}\left(M_{0}\right) \geq h_{1}\left(M_{0}\right) \alpha_{0}$, where $\alpha_{0}=\max _{x \in \bar{\Omega}} \int_{\Omega} \rho(\eta, x, y) d y$.

It is easily seen that Assumptions (A1)-(A5) are satisfied by $g(u)=\alpha u$ in the most population models and $f(u)=p u e^{-q u}$ in the Nicholson's blowflies model [14], where $p, q, \alpha>0$.

The rest of this paper is organized as follows. We present some preliminary results in Section 2. Our main results are presented and proved in Sections 3 and 4. In Section 3, we obtain some sufficient conditions for nonexistence, existence and uniqueness of positive equilibriums to (1.2) by employing the method of super-sub solutions. In Section 4, developed is a technique that combines the method of super-sub solutions, the variationof-constants formula for the delay differential equation and the estimation of integral kernels, which enables us to obtain some sufficient conditions for the global attractivity of the unique positive equilibrium. Finally, we present two examples in Section 5 to illustrate the obtained results.

Remark 1.1 If we let $\eta=0$, then (1.2) reduces to the following local time-delayed reactiondiffusion equation:

$$
\left\{\begin{array}{l}
\frac{\partial u(t, x)}{\partial t}=d \Delta u(t, x)-g(u(t, x))+f(u(t-\tau, x)), \quad t>0, x \in \Omega, \\
B u(t, x)=0, \quad t>0, x \in \partial \Omega \\
u(\theta, x)=\varphi(\theta, x), \quad \theta \in[-\tau, 0], x \in \Omega .
\end{array}\right.
$$

Yi and Zou [6] analyzed the special case of $B u=\partial u / \partial \mathbf{n}, g(u)=\alpha u$ and $f(u)=p u e^{-q u}$ in (1.4), where $\alpha, p, q>0$. They obtained some sufficient conditions for the global attractiveness of the unique positive steady state by combining a dynamical systems argument and some subtle inequalities [6].

Remark 1.2 If we let $d \rightarrow 0^{+}$in (1.2), then we obtain the following nonlocal time-delayed differential equation:

$$
\left\{\begin{array}{l}
\frac{\partial u(t, x)}{\partial t}=-g(u(t, x))+\int_{\bar{\Omega}} \rho(\eta, x, y) f(u(t-\tau, y)) d y, \quad t>0, x \in \Omega \\
B u(t, x)=0, \quad t>0, x \in \partial \Omega \\
u(\theta, x)=\varphi(\theta, x), \quad \theta \in[-\tau, 0], x \in \Omega
\end{array}\right.
$$

Yuan and Guo [9] considered this equation. By employing the method of super-sub solutions, combined with the careful analysis for $\rho(\eta, x, y)$, they proved nonexistence, existence and uniqueness of positive equilibriums to (1.5). With the help of the comparison principle, they employed the theory of dissipative systems to obtain the global asymptotic stability of the unique positive equilibrium to (1.5).

Remark 1.3 If we let $\eta=0$ and $d \rightarrow 0^{+}$, then (1.2) reduces to the following delay differential equation:

$$
\left\{\begin{array}{l}
\frac{d u(t)}{d t}=-g(u(t))+f(u(t-\tau)), \quad t>0, \\
u(\theta)=\varphi(\theta), \quad \theta \in[-\tau, 0] .
\end{array}\right.
$$

Its dynamics have been extensively and intensively studied for the case of $g(u)=\alpha u$, where $\alpha>0$; see, e.g., [14-24] and the references therein. 


\section{Preliminaries}

Let $\mathbb{Y}=C(\bar{\Omega}, \mathbb{R})$ and $\mathbb{Y}^{+}=\{\varphi \in \mathbb{Y} \mid \varphi(x) \geq 0$ for all $x \in \bar{\Omega}\}$. Then $\left(\mathbb{Y}, \mathbb{Y}^{+}\right)$is a strongly ordered Banach space. It is well known that the operator $d \Delta$ generates a $C^{0}$-semigroup $S(t)$ on $\mathbb{Y}$. Moreover, the standard parabolic maximum principle (see, e.g., Corollary 7.2.3 of [25]) implies that $S(t): \mathbb{Y} \rightarrow \mathbb{Y}$ is strongly positive in the sense that $S(t)\left(\mathbb{Y}^{+} \backslash\{0\}\right) \subset \operatorname{int}\left(\mathbb{Y}^{+}\right)$for all $t>0$.

Let $\mathbb{C}=C([-\tau, 0], \mathbb{Y})$ and $\mathbb{C}^{+}=C\left([-\tau, 0], \mathbb{Y}^{+}\right)$. For any continuous function $u(\cdot)$ : $[-\tau, \varrho) \rightarrow \mathbb{Y}$, where $\varrho>0$, define $u_{t} \in \mathbb{C}, t \in[0, \varrho)$ by $u_{t}(\theta)=u(t+\theta)$ for all $\theta \in[-\tau, 0]$ and its norm

$$
\left\|u_{t}\right\|_{\mathbb{C}}=\sup _{\theta \in[-\tau, 0]} \sup _{x \in \bar{\Omega}}|u(t+\theta, x)|
$$

where we denote $u(t, x)=u(t)(x), t \in[-\tau, \varrho), x \in \bar{\Omega}$. Define $G: \mathbb{C}^{+} \rightarrow \mathbb{Y}$ by

$$
G(\varphi)(x)=-g(\varphi(0, x))+\int_{\bar{\Omega}} \rho(\eta, x, y) f(\varphi(-\tau, y)) d y, \quad \forall x \in \bar{\Omega}, \varphi \in \mathbb{C}^{+} .
$$

Then equation (1.2) can be rewritten as the following integral equation:

$$
\left\{\begin{array}{l}
u(t)=S(t) \varphi(0)+\int_{0}^{t} S(t-s) G\left(u_{s}\right) d s, \quad t \geq 0 \\
u_{0}=\varphi \in \mathbb{C}^{+}
\end{array}\right.
$$

whose solution is called a mild solution to (1.2).

Since $S(t): \mathbb{Y} \rightarrow \mathbb{Y}$ is strongly positive, we obtain that

$$
\lim _{\varepsilon \rightarrow 0^{+}} \operatorname{dist}\left(\varphi(0)+\varepsilon G(\varphi), \mathbb{Y}^{+}\right)=0, \quad \forall \varphi \in \mathbb{C}^{+} .
$$

By [26] (Corollary 8.1.3) (see also Proposition 3 and Remark 2.4 of [27]), for any $\varphi \in \mathbb{C}^{+}$, equation (1.2) has a unique non-continuable mild solution $u(t, \varphi)$ with $u_{0}=\varphi$, and $u(t, \varphi) \in$ $\mathbb{Y}^{+}$for all $t \in\left(0, \varrho_{\varphi}\right)$. Moreover, $u(t, \varphi)$ is also a classical solution of (1.2) for all $t>\tau$ (see Corollary 2.2.5 of [26]).

By similar arguments as in the proof of [3] (Theorem 2.1), we obtain the following lemma.

Lemma 2.1 If (A1)-(A4) hold, then for any $\varphi \in \mathbb{C}^{+}$, a unique solution $u(t, \varphi)$ for (1.2) globally exists on $[-\tau, \infty), \limsup _{t \rightarrow+\infty} u(t, x, \varphi) \leq M$ uniformly for all $x \in \bar{\Omega}$, and the solution semiflow $\Psi(t)=u_{t}(\cdot): \mathbb{C}^{+} \rightarrow \mathbb{C}^{+}, t \geq 0$, admits a connected global attractor.

Next, we consider the following elliptic eigenvalue problem:

$$
\left\{\begin{array}{l}
\lambda u(x)=d \Delta u(x)-h_{2}(0) u(x)+h_{1}(0) \int_{\bar{\Omega}} u(y) \rho(\eta, x, y) d y, \quad x \in \Omega, \\
B u(x)=0, \quad x \in \partial \Omega
\end{array}\right.
$$

By similar arguments as in the proof of Theorem 7.6.1 in [25], it follows that the eigenvalue problem (2.2) has a principal eigenvalue $\xi_{0}$. In fact, one can easily determine $\xi_{0}$ as

$$
\xi_{0}=-d \xi_{1}-h_{2}(0)+h_{1}(0) e^{-\eta \xi_{1}}
$$


Similar to the arguments as in the proof of [3] (Theorem 3.1), we obtain the following threshold type results on the global attractivity of the zero solution and uniform persistence for (1.2).

Lemma 2.2 Let $\psi^{*} \in \operatorname{int}\left(\mathbb{Y}^{+}\right)$be fixed and (A1)-(A4) hold. For any $\varphi \in \mathbb{C}^{+}$, denote by $u(t, x, \varphi)$ or $u(t, \varphi)$ the solution to (1.2). Then the following statements are valid.

(i) If $h_{1}(0) e^{-\eta \xi_{1}}<d \xi_{1}+h_{2}(0)$, then $\lim _{t \rightarrow+\infty}\|u(t, \varphi)\|_{\mathbb{Y}}=0$ for all $\varphi \in \mathbb{C}^{+}$.

(ii) If $h_{1}(0) e^{-\eta \xi_{1}}>d \xi_{1}+h_{2}(0)$, then (1.2) admits at least one equilibrium solution $u^{*}$ with $u^{*}(x) \in(0, M]$ for all $x \in \Omega$, and there exists $\delta>0$ such that for any $\varphi \in \mathbb{C}^{+}$with $\varphi(0, \cdot) \not \equiv 0$, there is $t_{0}=t_{0}(\varphi)>0$ such that $u(t, x, \varphi) \geq \delta \psi^{*}(x)$ for all $x \in \bar{\Omega}$ and $t \geq t_{0}$.

\section{Existence and uniqueness of positive steady states}

In this section, we consider existence and uniqueness of positive equilibriums to (1.2). The equilibriums are also positive solutions to the following boundary value problem:

$$
\left\{\begin{array}{l}
-d \Delta u(x)+g(u(x))=\int_{\bar{\Omega}} \rho(\eta, x, y) f(u(y)) d y, \quad x \in \Omega \\
B u(x)=0, \quad x \in \partial \Omega
\end{array}\right.
$$

By the famous Krein-Rutman theorem [28] and similar arguments as in the proof of Theorem 2.3 in [9] (or Theorem 2.6 in [10]), we can obtain the following theorem which provides a sufficient condition for nonexistence of positive equilibriums to (1.2).

Theorem 3.1 If (A1) and (A3) hold, and

$$
h_{1}(0) e^{-\eta \xi_{1}} \leq d \xi_{1}+h_{2}(0)
$$

then (3.1) has no positive solution.

Next, we will employ a technique developed in [4] to analyze the existence and uniqueness of positive equilibriums to (1.2), that is, the existence and uniqueness of positive solutions to (3.1).

It is easy to see from Assumption (A2) that $f^{\prime}(u)$ is bounded from below. Thus, we can let $\tilde{\gamma}=\inf _{u \geq 0} f^{\prime}(u)$. Furthermore, we let $g_{0}(u)=\alpha u-g(u)$ and $f_{0}(u)=f(u)+\gamma u$, where $\alpha=\max _{u \in\left[0, M_{1}\right]} g^{\prime}(u), M_{1}=\max \left\{1+M, M_{0}\right\}$ and

$$
\gamma= \begin{cases}-\tilde{\gamma}, & \tilde{\gamma}<0 \\ 0, & \tilde{\gamma} \geq 0\end{cases}
$$

So, $f_{0}^{\prime}(u) \geq 0$ for all $u \geq 0$. Define $F: \mathbb{Y} \rightarrow \mathbb{Y}$ by

$$
F(u)(x)=g_{0}(u(x))+\int_{\bar{\Omega}} \rho(\eta, x, y) f_{0}(u(y)) d y, \quad \forall x \in \bar{\Omega}, u \in \mathbb{Y}
$$

Then boundary value problem (3.1) can be rewritten as

$$
\left\{\begin{array}{l}
-d \Delta u(x)+\alpha u(x)+\gamma \int_{\bar{\Omega}} \rho(\eta, x, y) u(y) d y=F(u)(x), \quad x \in \Omega, \\
B u(x)=0, \quad x \in \partial \Omega .
\end{array}\right.
$$


For any constant $C$, we always denote by $\widehat{C}$ a constant function on $\bar{\Omega}$ taking the value $C$. Then, by Lemma 2.3 of [10], the operator $F$ is positive and monotone in the order interval $\left[\widehat{0}, \widehat{M_{1}}\right]$. Now, we consider the following integral equation:

$$
\left\{\begin{array}{l}
u(x)=\int_{\bar{\Omega}} k(\eta, x, y) F(u)(y) d y, \quad x \in \Omega \\
B u(x)=0, \quad x \in \partial \Omega
\end{array}\right.
$$

where

$$
k(\eta, x, y)=\sum_{n=1}^{+\infty} \frac{1}{d \xi_{n}+\alpha+\gamma e^{-\eta \xi_{n}}} \psi_{n}(x) \psi_{n}(y)
$$

Motivated by [10] (Section 2), we assume that the constant $\gamma$ satisfies (A6)

$$
\gamma \in \begin{cases}\left\{\lambda \in \mathbb{R} \mid 0 \leq \lambda \leq \frac{d}{\eta} e^{-\left(\frac{\alpha \eta}{d}+1\right)}\right\}, & \text { if } \eta>0, \\ \{\lambda \in \mathbb{R} \mid 0 \leq \lambda<+\infty\}, & \text { if } \eta=0,\end{cases}
$$

where $\gamma \in$ denotes the real number $\gamma$ belonging to the next set of real numbers. By similar arguments as in the proof of [10] (Lemmas 2.7, 2.8 and Theorem 2.9), we obtain the following results.

Lemma 3.1 If $u \in \mathbb{Y}$ is a solution to (3.5), then it is also a solution to (3.6) and vice versa.

Lemma 3.2 If (A6) holds, then $k(\eta, x, y)>0$ for all $x, y \in \Omega$.

Theorem 3.2 If (A1)-(A6) hold, and

$$
d \xi_{1}+h_{2}(0)<h_{1}(0) e^{-\eta \xi_{1}}
$$

then (3.1) has a unique positive solution.

\section{Global attractivity}

In this section, we establish the global attractivity of the positive equilibrium $u^{*}$ to (1.2) by developing a new approach.

Lemma 4.1 Assume that (A1)-(A4) hold, and let $u(t, x) \equiv u(t, x, \varphi)$ be the solution to (1.2) with $\varphi \in \mathbb{C}^{+}$. Then $u(t, x)$ satisfies

$$
\begin{aligned}
u(t, x)= & \int_{\Omega} \mathcal{K}(t, x, y) \varphi(0, y) d y+\int_{0}^{t} \int_{\Omega} \mathcal{K}_{1}(s, x, y) \varphi(t-s-\tau, y) d y d s \\
& +\int_{0}^{t} \int_{\Omega} \mathcal{K}(s, x, y) F(u)(t-s, y) d y d s, \quad \forall t>\tau, x \in \Omega .
\end{aligned}
$$

Here, we have extended $\varphi(t)$ to $[-\tau, \infty)$ by making it zero for $t>0$. The real numbers $\alpha$ and $\gamma$ are defined in Section 3. The operator F is given in (3.4), that is, for any $t>0$,

$$
F(u)(t, x)=g_{0}(u(t, x))+\int_{\bar{\Omega}} \rho(\eta, x, y) f_{0}(u(t-\tau, y)) d y, \quad \forall u \in \mathbb{Y}, x \in \Omega
$$


And the kernel functions $\mathcal{K}(t, x, y)$ and $\mathcal{K}_{1}(t, x, y)$ are given by

$$
\mathcal{K}(t, x, y)=\sum_{n=1}^{+\infty} K_{n}(t) e^{-\left(\alpha+d \xi_{n}\right) t} \psi_{n}(x) \psi_{n}(y), \quad \forall x, y \in \Omega,
$$

and

$$
\mathcal{K}_{1}(t, x, y)=\sum_{n=1}^{+\infty} \widetilde{\gamma}_{n} K_{n}(t) e^{-\left(\alpha+d \xi_{n}\right)(t+\tau)} \psi_{n}(x) \psi_{n}(y), \quad \forall x, y \in \Omega
$$

where

$$
K_{n}(t)=\sum_{j=0}^{l} \frac{1}{j !} \widetilde{\gamma}_{n}^{j}(t-j \tau)^{j}, \quad l \tau \leq t<(l+1) \tau, l=0,1,2, \ldots
$$

and

$$
\widetilde{\gamma}_{n}=-\gamma e^{-\eta \xi_{n}} e^{\left(\alpha+d \xi_{n}\right) \tau}
$$

Proof Since $\mathbb{Y} \subset L^{2}(\bar{\Omega})$, for each $t \geq 0$, there exist real numbers $a_{n}(t)$ and $b_{n}(t), n=1,2, \ldots$, such that

$$
u(t, x)=\sum_{n=1}^{+\infty} a_{n}(t) \psi_{n}(x)
$$

and

$$
F(u)(t, x)=\sum_{n=1}^{+\infty} b_{n}(t) \psi_{n}(x)
$$

Therefore, by (4.7), (4.8) and (1.2), we have

$$
\begin{aligned}
& a_{n}(0)=\int_{\Omega} \varphi(0, y) \psi_{n}(y) d y, \\
& b_{n}(t)=\int_{\Omega} F(u)(t, y) \psi_{n}(y) d y
\end{aligned}
$$

and

$$
a_{n}^{\prime}(t)=-\left(\alpha+d \xi_{n}\right) a_{n}(t)-\gamma e^{-\eta \xi_{n}} a_{n}(t-\tau)+b_{n}(t), \quad n=1,2, \ldots
$$

By using the variation-of-constants formula for the nonhomogeneous linear delay differential equation (see, e.g., Section 4.2 of [29]), we obtain

$$
\begin{aligned}
a_{n}(t)= & e^{-\left(\alpha+d \xi_{n}\right) t}\left(K_{n}(t) a_{n}(0)+\widetilde{\gamma}_{n} \int_{0}^{t} K_{n}(s) \varphi_{n}(t-s-\tau) d s\right) \\
& +\int_{0}^{t} K_{n}(s) b_{n}(t-s) e^{-\left(\alpha+d \xi_{n}\right) s} d s, \quad t>\tau, n=1,2, \ldots
\end{aligned}
$$


Here, $K_{n}(t)$ and $\tilde{\gamma}_{n}$ are given in (4.5) and (4.6), respectively, and

$$
\varphi_{n}(t)= \begin{cases}e^{\left(\alpha+d \xi_{n}\right) t} \int_{\Omega} \varphi(t, y) \psi_{n}(y) d y, & t \in[-\tau, 0] \\ 0, & t>0 .\end{cases}
$$

Thus, by (4.7), (4.9), (4.10), (4.12) and (4.13), we have

$$
\begin{aligned}
u(t, x)= & \sum_{n=1}^{+\infty} e^{-\left(\alpha+d \xi_{n}\right) t}\left(K_{n}(t) a_{n}(0)+\tilde{\gamma}_{n} \int_{0}^{t} K_{n}(s) \varphi_{n}(t-s-\tau) d s\right) \psi_{n}(x) \\
& +\sum_{n=1}^{+\infty} \psi_{n}(x) \int_{0}^{t} K_{n}(s) b_{n}(t-s) e^{-\left(\alpha+d \xi_{n}\right) s} d s \\
= & \int_{\Omega}\left[\sum_{n=1}^{+\infty} K_{n}(t) e^{-\left(\alpha+d \xi_{n}\right) t} \psi_{n}(x) \psi_{n}(y)\right] \varphi(0, y) d y \\
& +\int_{0}^{t} \int_{\Omega}\left[\sum_{n=1}^{+\infty} \tilde{\gamma}_{n} K_{n}(s) e^{-\left(\alpha+d \xi_{n}\right)(s+\tau)} \psi_{n}(x) \psi_{n}(y)\right] \varphi(t-s-\tau, y) d y d s \\
& +\int_{0}^{t} \int_{\Omega}\left[\sum_{n=1}^{+\infty} K_{n}(s) e^{-\left(\alpha+d \xi_{n}\right) s} \psi_{n}(x) \psi_{n}(y)\right] F(u)(t-s, y) d y d s, \quad t>\tau .
\end{aligned}
$$

Therefore, (4.1) follows immediately from (4.3), (4.4) and (4.14). The proof is completed.

Lemma 4.2 Let

$$
\mathfrak{L}_{l}(\theta)=\sum_{j=0}^{l} \frac{1}{j !}\left(-\gamma_{1}\right)^{j}(l-j+\theta)^{j}
$$

where $\gamma_{1}>0, \theta \in[0,1]$ and $l=0,1,2, \ldots$. If $\gamma_{1} \leq e^{-1}$, then $\mathfrak{L}_{l}(\theta)>0$.

Proof Let

$$
\Gamma_{l}(\theta)=e^{\theta} \mathfrak{L}_{l}(\theta), \quad l=0,1,2, \ldots
$$

Then $\Gamma_{0}(\theta)=e^{\theta}$ is positive and monotone nondecreasing in the interval $[0,1]$. Assume that $\Gamma_{l}(\theta)$ is positive and monotone nondecreasing for all $\theta \in[0,1]$. Next, we will prove that $\Gamma_{l+1}(\theta)$ is positive and monotone nondecreasing for all $\theta \in[0,1]$. In fact, since

$$
\Gamma_{l+1}(0)=\mathfrak{L}_{l+1}(0)=\mathfrak{L}_{l}(1)=e^{-1} \Gamma_{l}(1)
$$

and

$$
\left(\Gamma_{l+1}(\theta) e^{-\theta}\right)^{\prime}=\mathfrak{L}_{l+1}^{\prime}(\theta)=-\gamma_{1} \mathfrak{L}_{l}(\theta)=-\gamma_{1} e^{-\theta} \Gamma_{l}(\theta),
$$

we have

$$
\Gamma_{l+1}(\theta) e^{-\theta}=e^{-1} \Gamma_{l}(1)-\gamma_{1} \int_{0}^{\theta} e^{-s} \Gamma_{l}(s) d s,
$$


that is,

$$
\Gamma_{l+1}(\theta)=e^{-1+\theta} \Gamma_{l}(1)-\gamma_{1} e^{\theta} \int_{0}^{\theta} e^{-s} \Gamma_{l}(s) d s .
$$

It follows from (4.18) and the monotonicity of $\Gamma_{l}(\theta)$ that

$$
\begin{aligned}
\Gamma_{l+1}^{\prime}(\theta) & =\Gamma_{l+1}(\theta)-\gamma_{1} \Gamma_{l}(\theta) \\
& =e^{-1+\theta} \Gamma_{l}(1)-\gamma_{1} e^{\theta} \int_{0}^{\theta} e^{-s} \Gamma_{l}(s) d s-\gamma_{1} \Gamma_{l}(\theta) \\
& \geq e^{-1+\theta} \Gamma_{l}(1)-\gamma_{1} e^{\theta} \Gamma_{l}(1) \int_{0}^{\theta} e^{-s} d s-\gamma_{1} \Gamma_{l}(1) \\
& =\Gamma_{l}(1)\left[e^{-1+\theta}-\gamma_{1} e^{\theta}\left(1-e^{-\theta}\right)-\gamma_{1}\right] \\
& =e^{\theta} \Gamma_{l}(1)\left[e^{-1}-\gamma_{1}\right] \\
& \geq 0 .
\end{aligned}
$$

Thus, $\Gamma_{l+1}(\theta)$ increases with $\theta \in[0,1]$. Moveover, by (4.17), we know that $\Gamma_{l+1}(\theta)>0$ for all $\theta \in[0,1]$. It follows from induction that $\Gamma_{l}(\theta)>0$ for all $\theta \in[0,1]$ and $l=0,1,2, \ldots$. So, $\mathfrak{L}_{l}(\theta)>0$ for all $\theta \in[0,1]$ and $l=0,1,2, \ldots$. The proof is completed.

Lemma 4.3 Assume that $\mathcal{K}(s, x, y)$ and $k(\eta, x, y)$ are given by (4.3) and (3.7), respectively. Then

$$
\int_{0}^{+\infty} \mathcal{K}(s, x, y) d s=k(\eta, x, y) .
$$

Proof By (4.3), we have

$$
\mathcal{K}(s, x, y)=\sum_{n=1}^{+\infty} \sum_{j=0}^{l} \frac{1}{j !}(-\gamma)^{j} e^{-\eta \xi_{n} j} e^{\left(\alpha+d \xi_{n}\right) \tau j}(s-j \tau)^{j} e^{-\left(\alpha+d \xi_{n}\right) s} \psi_{n}(x) \psi_{n}(y),
$$

where $l \tau \leq s<(l+1) \tau, l=0,1,2, \ldots$. Let

$$
c_{l j}=\sum_{i=0}^{j} \frac{j !(l-j)^{j-i} \tau^{j-i}}{(j-i) !\left(\alpha+d \xi_{n}\right)^{i+1}} e^{-\left(\alpha+d \xi_{n}\right) l \tau}
$$

where $l, j=0,1,2, \ldots$. Then

$$
\int_{l \tau}^{(l+1) \tau}(s-j \tau)^{j} e^{-\left(\alpha+d \xi_{n}\right) s} d s=c_{l j}-c_{l+1, j}
$$

It follows from (4.20) and (4.22) that

$$
\begin{aligned}
\int_{0}^{+\infty} \mathcal{K}(s, x, y) d s & =\sum_{l=0}^{+\infty} \int_{l \tau}^{(l+1) \tau} \mathcal{K}(s, x, y) d s \\
& =\sum_{n=1}^{+\infty} \sum_{l=0}^{+\infty} \sum_{j=0}^{l} \frac{1}{j !}(-\gamma)^{j} e^{-\eta \xi_{n} j} e^{\left(\alpha+d \xi_{n}\right) \tau j}\left(c_{l j}-c_{l+1, j}\right) \psi_{n}(x) \psi_{n}(y)
\end{aligned}
$$




$$
\begin{aligned}
& =\sum_{n=1}^{+\infty} \sum_{l=0}^{+\infty} \sum_{j=l}^{+\infty} \frac{1}{l !}(-\gamma)^{l} e^{-\eta \xi_{n} l} e^{\left(\alpha+d \xi_{n}\right) \tau l}\left(c_{j l}-c_{j+1, l}\right) \psi_{n}(x) \psi_{n}(y) \\
& =\sum_{n=1}^{+\infty} \sum_{l=0}^{+\infty} \frac{1}{l !}(-\gamma)^{l} e^{-\eta \xi_{n} l} e^{\left(\alpha+d \xi_{n}\right) \tau l} c_{l l} \psi_{n}(x) \psi_{n}(y) \\
& =\sum_{n=1}^{+\infty} \sum_{l=0}^{+\infty} \frac{1}{\left(\alpha+d \xi_{n}\right)^{l+1}}(-\gamma)^{l} e^{-\eta \xi_{n} l} \psi_{n}(x) \psi_{n}(y) \\
& =\sum_{n=1}^{+\infty} \frac{1}{\alpha+d \xi_{n}+\gamma e^{-\eta \xi_{n}}} \psi_{n}(x) \psi_{n}(y) \\
& =k(\eta, x, y) .
\end{aligned}
$$

The proof is completed.

\section{Lemma 4.4 If}

$$
\gamma \leq \tau^{-1} e^{-(\alpha \tau+1)}
$$

and $\eta=d \tau$, then

$$
\mathcal{K}(t, x, y)>0
$$

for all $t \in(0,+\infty)$ and $x, y \in \Omega$.

Proof Let $t=(l+\theta) \tau$. Then, by (4.5), we have $\theta \in[0,1)$. By (4.3), (4.15) and $\eta=d \tau$, we obtain that

$$
\begin{aligned}
\mathcal{K}(t, x, y) & =\sum_{n=1}^{+\infty} \sum_{j=0}^{l} \frac{1}{j !}(-\gamma)^{j} e^{-\eta j \xi_{n}} e^{-\left(\alpha+d \xi_{n}\right)(t-j \tau)}(t-j \tau)^{j} \psi_{n}(x) \psi_{n}(y) \\
& =e^{-\alpha t} \sum_{j=0}^{l} \frac{1}{j !}(-\gamma)^{j} e^{\alpha \tau j}(t-j \tau)^{j} \sum_{n=1}^{+\infty} e^{-d t \xi_{n}} \psi_{n}(x) \psi_{n}(y) \\
& =e^{-\alpha t} \sum_{j=0}^{l} \frac{1}{j !}\left(-\gamma \tau e^{\alpha \tau}\right)^{j}(l-j+\theta)^{j} \sum_{n=1}^{+\infty} e^{-d t \xi_{n}} \psi_{n}(x) \psi_{n}(y) \\
& =e^{-\alpha t} \sum_{j=0}^{l} \frac{1}{j !}\left(-\gamma_{1}\right)^{j}(l-j+\theta)^{j} \sum_{n=1}^{+\infty} e^{-d t \xi_{n}} \psi_{n}(x) \psi_{n}(y) \\
& =e^{-\alpha t} \mathfrak{L}_{l}(\theta) \sum_{n=1}^{+\infty} e^{-d t \xi_{n}} \psi_{n}(x) \psi_{n}(y),
\end{aligned}
$$

where $\gamma_{1}=\gamma \tau e^{\alpha \tau}$. Thus, (4.25) follows immediately from (4.24), (4.26) and Lemma 4.2. The proof is completed.

By Lemma 4.4, we have the following lemma.

Lemma 4.5 If (4.24) holds, then there exist two constants $\bar{\varepsilon}>0$ and $\underline{\varepsilon}>0$ such that (4.25) holds for all $-\underline{\varepsilon}<\eta-d \tau<\bar{\varepsilon}$. 
Let

$$
\mathfrak{L}_{l n}(\theta, \varepsilon)=\sum_{j=0}^{l} \frac{1}{j !}\left(-\gamma \tau e^{\alpha \tau}\right)^{j}(l-j+\theta)^{j} e^{-(\varepsilon j+d \tau(l+\theta)) \xi_{n}},
$$

where $\theta \in[0,1), \varepsilon \in \mathbb{R}, n=1,2, \ldots$ and $l=0,1,2, \ldots$ By Lemma 4.5 , we can define

$$
\bar{\varepsilon}_{0}=\sup \left\{\varepsilon \in \mathbb{R}^{+} \mid \sum_{n=1}^{+\infty} \mathfrak{L}_{l n}(\theta, \varepsilon) \psi_{n}(x) \psi_{n}(y)>0, \forall x, y \in \Omega, l \in \mathbb{N}, \theta \in[0,1)\right\}
$$

and

$$
\underline{\varepsilon}_{0}=\sup \left\{\varepsilon \in \mathbb{R}^{+} \mid \sum_{n=1}^{+\infty} \mathfrak{L}_{l n}(\theta,-\varepsilon) \psi_{n}(x) \psi_{n}(y)>0, \forall x, y \in \Omega, l \in \mathbb{N}, \theta \in[0,1)\right\},
$$

where $\mathbb{N}$ is the set of natural numbers. Furthermore, we assume that

(A7) $\gamma$ and $\eta$ satisfy (4.24) and $d \tau-\underline{\varepsilon}_{0}<\eta<d \tau+\bar{\varepsilon}_{0}$, respectively.

Then, by Lemmas 4.3 and 4.5 , we have the following lemma.

Lemma 4.6 If (A7) holds, then $\mathcal{K}(t, x, y)>0$ and $k(\eta, x, y)>0$ for all $t>0$ and $x, y \in \Omega$.

Next, we prove our main result in this section.

Theorem 4.1 Assume that $h_{1}(0) e^{-\eta \xi_{1}}>d \xi_{1}+h_{2}(0)$. If (A1)-(A5) and (A7) hold, then (1.2) admits a unique positive equilibrium $u^{*}$ and

$$
\lim _{t \rightarrow+\infty}\left\|u(t, \varphi)-u^{*}\right\|_{\mathbb{Y}}=0
$$

for every $\varphi \in \mathbb{C}^{+}$with $\varphi(0, \cdot) \not \equiv 0$, where $u(t, \varphi)$ is the solution to (1.2).

Proof By Lemma 4.6 and Theorem 3.2, we know that (1.2) has a unique positive equilibrium $u^{*}$. By Lemma 2.1, it is sufficient to prove that the unique positive equilibrium $u^{*}$ is globally attractive in $\mathbb{C}_{[0, M+1]} \backslash\{0\}$, where

$$
\mathbb{C}_{[0, M+1]}=\left\{\varphi \in \mathbb{C}^{+} \mid 0 \leq \varphi(\theta, x) \leq M+1, \forall(\theta, x) \in[-\tau, 0] \times \bar{\Omega}\right\}
$$

For any given $\varphi \in \mathbb{C}_{[0, M+1]} \backslash\{0\}$, (4.1) holds. Let

$$
u^{\infty}(x) \equiv \limsup _{t \rightarrow \infty} u(t, x), \quad u_{\infty}(x) \equiv \liminf _{t \rightarrow \infty} u(t, x), \quad \forall x \in \bar{\Omega}
$$

Then, by Lemmas 2.1 and 2.2, there exists a constant $\delta>0$ such that

$$
M+1 \geq u^{\infty}(x) \geq u_{\infty}(x) \geq \delta \psi^{*}(x)>0, \quad \forall x \in \Omega,
$$


where $\psi^{*}$ is given in Lemma 2.2. On the other hand, note that $F(u)$ is nondecreasing in $u \in[0, M+1]$. Thus, by Fatou's lemma, Lemmas 4.3 and 4.6, we further get

$$
\begin{aligned}
u^{\infty}(x) & \leq \int_{0}^{\infty} \int_{\Omega} \mathcal{K}(s, x, y) F\left(u^{\infty}(y)\right) d y d s \\
& =\int_{\Omega} k(\eta, x, y) F\left(u^{\infty}(y)\right) d y .
\end{aligned}
$$

Thus, $u^{\infty}(x)$ is a sub-solution to (3.6). Similarly, $u_{\infty}(x)$ is a super-solution to (3.6). Let $\underline{u}(x)=\epsilon \psi_{1}(x)$ and $\bar{u}(x)=\widehat{M_{1}}$, where $M_{1}$ is given in Section 3 and $\epsilon$ is a sufficiently small positive number such that $\epsilon \psi_{1}(x) \leq \delta \psi^{*}(x)$ for all $x \in \Omega$. By similar arguments as in the proof of [10] (Theorem 2.9), we obtain that $\underline{u}$ and $\bar{u}$ are the sub-solution and super-solution of (3.6), respectively. Furthermore, by employing a standard super- and sub-solution argument, we know that (3.6) has at least one positive solution $\underline{u}^{*}$ in the order interval $\left[\underline{u}, u_{\infty}\right]$. Similarly, (3.6) admits at least one positive solution $\bar{u}^{*}$ in the order interval $\left[u^{\infty}, \bar{u}\right]$. By Lemmas 3.1, 4.6 and Theorem 3.2, $\underline{u}^{*}=\bar{u}^{*}=u^{*}$. Thus, $u_{\infty}=u^{\infty}=u^{*}$. This implies that

$$
\lim _{t \rightarrow+\infty} u(t, x)=u^{*}(x), \quad \forall x \in \bar{\Omega}
$$

It remains to prove that $\lim _{t \rightarrow+\infty} u(t, x)=u^{*}(x)$ uniformly for $x \in \bar{\Omega}$. For any $\phi \in \omega(\varphi)$, there exists a sequence $t_{n} \rightarrow+\infty$ such that $\Psi\left(t_{n}\right) \varphi \rightarrow \phi$ in $\mathbb{C}_{[0, M+1]}$ as $n \rightarrow \infty$. Then we obtain that

$$
\lim _{n \rightarrow \infty} u\left(t_{n}+\theta, x, \varphi\right)=\phi(\theta, x)
$$

uniformly for $(\theta, x) \in[-\tau, 0] \times \bar{\Omega}$. It follows from (4.32) that $\phi(\theta, x)=u^{*}(x)$ for all $(\theta, x) \in$ $[-\tau, 0] \times \bar{\Omega}$. Thus, we obtain that $\omega(\varphi)=\left\{u^{*}\right\}$, which implies that $u(t, \cdot, \varphi)$ converges to $u^{*}$ in $\mathbb{Y}$ as $t \rightarrow+\infty$. The proof is completed.

\section{Examples}

In this section, we present two examples to illustrate our main results.

First we consider the following local Nicholson's blowfly equation:

$$
\left\{\begin{array}{l}
\frac{\partial u(t, x)}{\partial t}=d \Delta u(t, x)-\alpha u(t, x)+f_{1}(u(t-\tau, x)), \quad t>0, x \in \Omega, \\
\frac{\partial}{\partial \mathbf{n}} u(t, x)=0, \quad t>0, x \in \partial \Omega \\
u(\theta, x)=\phi(\theta, x), \quad \theta \in[-\tau, 0], x \in \Omega .
\end{array}\right.
$$

Here, $d>0, \alpha>0$ and $f_{1}(w)=\varepsilon p u e^{-q u}$ which is referred to as Ricker's birth function in population dynamics (see, e.g., [5-11,30-33]), where $\varepsilon>0, p>0$ and $q>0$.

By Lemma 2.2, Theorems 3.1, 3.2 and 4.1, we have the following.

\section{Proposition 5.1}

(i) If $\varepsilon p \leq \alpha$, then (5.1) has no positive steady state.

(ii) If $\varepsilon p>\alpha$, then (5.1) has a unique positive steady state $u^{*}$. 


\section{Proposition 5.2}

(i) If $\varepsilon p<\alpha$, then $\lim _{t \rightarrow+\infty}\|u(t, \varphi)\|_{\mathbb{Y}}=0$ for every $\varphi \in \mathbb{C}^{+}$, where $u(t, \phi)$ is the solution to $(5.1)$.

(ii) If $\alpha<\varepsilon p \leq \tau^{-1} e^{1-\alpha \tau}$ and $0<d<d_{0}$, where

$$
\begin{aligned}
& d_{0}=\sup \left\{\lambda \in \mathbb{R}^{+} \mid \sum_{n=1}^{+\infty} \mathfrak{L}_{0 l n}(\lambda, \theta) \psi_{n}(x) \psi_{n}(y)>0, \forall x, y \in \Omega, l \in \mathbb{N}, \theta \in[0,1)\right\}, \\
& \mathfrak{L}_{0 l n}(\lambda, \theta)=\sum_{j=0}^{l} \frac{1}{j !}\left(-\varepsilon p \tau e^{\alpha \tau-2}\right)^{j}(l+\theta-j)^{j} e^{-\lambda \tau(l+\theta-j) \xi_{n}}
\end{aligned}
$$

and $\mathbb{N}$ is the set of natural numbers, then (5.1) admits a unique positive steady state $u^{*}$ and $\lim _{t \rightarrow+\infty}\left\|u(t, \varphi)-u^{*}\right\|_{\mathbb{Y}}=0$ for every $\varphi \in \mathbb{C}^{+}$with $\varphi(0, \cdot) \not \equiv$.

Remark 5.1 When $\tau \rightarrow 0^{+}$, (5.1) reduces to the following local reaction-diffusion equation:

$$
\left\{\begin{array}{l}
\frac{\partial u(t, x)}{\partial t}=d \Delta u(t, x)-\alpha u(t, x)+f_{1}(u(t, x)), \quad t>0, x \in \Omega, \\
\frac{\partial}{\partial \mathbf{n}} u(t, x)=0, \quad t>0, x \in \partial \Omega, \\
u(0, x)=\varphi(x), \quad x \in \Omega .
\end{array}\right.
$$

By Propositions 5.1 and 5.2, we obtain a threshold type result, that is, if $\varepsilon p<\alpha$, then $\lim _{t \rightarrow+\infty}\|u(t, \varphi)\|_{\mathbb{Y}}=0$ for every $\varphi \in \mathbb{Y}^{+}$, and if $\varepsilon p>\alpha$, then (5.4) admits a unique positive steady state $u^{*}$ and $\lim _{t \rightarrow+\infty}\left\|u(t, \varphi)-u^{*}\right\|_{\mathbb{Y}}=0$ for every $\varphi \in \mathbb{Y}^{+}$with $\varphi(\cdot) \not \equiv 0$, where $u(t, \varphi)$ is the solution to (5.4).

The second example is the following nonlocal Mackey-Glass equation:

$$
\left\{\begin{aligned}
\frac{\partial u(t, x)}{\partial t}= & d \frac{\partial^{2} u(t, x)}{\partial x^{2}}-\alpha u(t, x) \\
& +\int_{0}^{\pi} \rho^{D}(\eta, x, y) f_{2}(u(t-\tau, y)) d y, \quad t>0, x \in(0, \pi), \\
u(t, 0)= & u(t, \pi)=0, \quad t>0, \\
u(\theta, x)= & \varphi(\theta, x), \quad \theta \in[-\tau, 0], x \in(0, \pi) .
\end{aligned}\right.
$$

Here, $d>0, \alpha>0, \eta>0, f_{2}(u)=\frac{p u}{q+u^{\beta}}, p>0, q>0, \beta>0$ and

$$
\rho^{D}(\eta, x, y)=\frac{2}{\pi} \sum_{n=1}^{+\infty} e^{-n^{2} \eta} \sin n x \sin n y
$$

This nonlinear function $f_{2}(u)$ was used as the production function for blood cells in [34], and has since been widely adapted; see, e.g., [7-11, 30,33] and the references therein.

By Lemma 2.2, Theorems 3.1, 3.2 and 4.1, we have the following.

\section{Proposition 5.3}

(i) If $\frac{p}{q} \leq(d+\alpha) e^{\eta}$, then (5.5) has no positive steady state. 
(ii) If

$$
\frac{p}{q} \in \begin{cases}\left\{\lambda \in \mathbb{R} \mid(d+\alpha) e^{\eta}<\lambda<+\infty\right\}, & \text { if } 0<\beta \leq 1, \\ \left\{\lambda \in \mathbb{R} \mid(d+\alpha) e^{\eta}<\lambda \leq \frac{4 d \beta}{\eta(\beta-1)^{2}} e^{-\left(\frac{\eta}{d} \alpha+1\right)}\right\}, & \text { if } \beta>1,\end{cases}
$$

then (5.5) has a unique positive steady state $u^{*}$.

\section{Proposition 5.4}

(i) If $\frac{p}{q}<(d+\alpha) e^{\eta}$, then $\lim _{t \rightarrow+\infty}\|u(t, \varphi)\|_{\mathbb{Y}}=0$ for every $\varphi \in \mathbb{C}^{+}$, where $u(t, \varphi)$ is the solution to (5.5).

(ii) Let

$$
\begin{gathered}
\underline{\varepsilon}_{1}=\sup \left\{\varepsilon \in \mathbb{R}^{+} \mid \sum_{n=1}^{+\infty} \mathfrak{L}_{1 l n}(\theta,-\varepsilon) \sin n x \sin n y>0,\right. \\
\forall x, y \in[0, \pi], l \in \mathbb{N}, \theta \in[0,1)\}, \\
\bar{\varepsilon}_{1}=\sup \left\{\varepsilon \in \mathbb{R}^{+} \mid \sum_{n=1}^{+\infty} \mathfrak{L}_{1 l n}(\theta, \varepsilon) \sin n x \sin n y>0,\right. \\
\forall x, y \in[0, \pi], l \in \mathbb{N}, \theta \in[0,1)\},
\end{gathered}
$$

where

$$
\mathfrak{L}_{1 l n}(\theta, \varepsilon)=\sum_{j=0}^{l} \frac{1}{j !}(-\tau p)^{j}(l+\theta-j)^{j}(\beta-1)^{2 j}(4 q \beta)^{-j} e^{\alpha \tau j} e^{-(\varepsilon j+d \tau(l+\theta)) n^{2}}
$$

and $\mathbb{N}$ is the set of natural numbers. If

$$
\frac{p}{q} \in \begin{cases}\left\{\lambda \in \mathbb{R} \mid(d+\alpha) e^{\eta}<\lambda<+\infty\right\}, & \text { if } 0<\beta \leq 1, \\ \left\{\lambda \in \mathbb{R} \mid(d+\alpha) e^{\eta}<\lambda \leq \frac{4 \beta}{\tau(\beta-1)^{2}} e^{-(\alpha \tau+1)}\right\}, & \text { if } \beta>1,\end{cases}
$$

and

$$
\eta \in \begin{cases}\mathbb{R}, & \text { if } 0<\beta \leq 1, \\ \left\{\lambda \in \mathbb{R} \mid d \tau-\underline{\varepsilon}_{1}<\lambda<d \tau+\bar{\varepsilon}_{1}\right\}, & \text { if } \beta>1\end{cases}
$$

then (5.5) admits a unique positive steady state $u^{*}$ and $\lim _{t \rightarrow+\infty}\left\|u(t, \varphi)-u^{*}\right\|_{\mathbb{Y}}=0$ for every $\varphi \in \mathbb{C}^{+}$with $\varphi(0, \cdot) \not \equiv 0$.

\section{Conclusion}

In this paper, we have proved the nonexistence, existence, uniqueness and global attractivity of positive steady state for (1.1). Our results extend almost all the related existing studies on the non-monotone time-delayed reaction-diffusion equations. In particular, 
developed is a technique that combines the method of super-sub solutions, the variationof-constants formula for the delay differential equation and the estimation of integral kernels, which enables us to obtain some sufficient conditions for the global attractiveness of the unique positive steady state for (1.1). Furthermore, it seems that the above technique can also be applied to the case of general delayed differential systems. Thus, the results obtained in this paper are interesting, and the approaches used to prove the main results are novel. In the future, we will consider the global dynamics for a large class of delayed differential systems with spatial non-locality by using the above technique.

\section{Acknowledgements}

The first author was partially supported by the National Natural Science Foundation of China (No. 11561068) and China Postdoctoral Science Foundation (2016M592442). The second author was partially supported by the National Natural Science Foundation of China (No. 11671403) and Mathematics and Interdisciplinary Sciences project of CSU.

\section{Competing interests}

The authors declare that they have no competing interests.

\section{Authors' contributions}

All authors contributed equally to the writing of this paper. All authors read and approved the final manuscript.

\section{Publisher's Note}

Springer Nature remains neutral with regard to jurisdictional claims in published maps and institutional affiliations.

Received: 23 October 2017 Accepted: 16 January 2018 Published online: 13 February 2018

\section{References}

1. Metz, JAJ, Diekmann, O (eds.): The Dynamics of Physiologically Structured Populations. Springer, New York (1986)

2. Gourley, SA, Wu, J: Delayed non-local diffusive systems in biological invasion and disease spread. In: Brunner, H, Zhao, X-Q, Zou, X (eds.) Nonlinear Dynamics and Evolution Equations. Fields Inst. Commun., vol. 48, pp. 137-200 (2006)

3. Xu, D, Zhao, X-Q: A nonlocal reaction-diffusion population model with stage structure. Can. Appl. Math. Q. 11, 303-319 (2003)

4. Guo, Z, Yang, ZC, Zou, X: Existence and uniqueness of positive solution to a non-local differential equation with homogeneous Dirichlet boundary condition: a non-monotone case. Commun. Pure Appl. Anal. 11, 1825-1838 (2012)

5. Liang, D, So, JW-H, Zhang, F, Zou, X: Population dynamic models with nonlocal delay on bounded fields and their numerical computations. Differ. Equ. Dyn. Syst. 11, 117-139 (2003)

6. Yi, T, Zou, X: Global attractivity of the diffusive Nicholson blowflies equation with Neumann boundary condition: a nonmonotone case. J. Differ. Equ. 245, 3376-3388 (2008)

7. Yi, T, Zou, X: On Dirichlet problem for a class of delayed reaction-diffusion equations with spatial non-locality. J. Dyn. Differ. Equ. 25, 959-979 (2013)

8. Yuan, Y, Guo, Z: Global asymptotic stability in a class of reaction-diffusion equations with time delay. Abstr. Appl. Anal. 2014, Article ID 378172 (2014)

9. Yuan, Y, Guo, Z: Global dynamics of a nonlocal population model with age structure in a bounded domain: a non-monotone case. Sci. China Math. 58, 2145-2166 (2015)

10. Yuan, Y, Guo, Z, Tang, M: A nonlocal diffusion population model with age structure and Dirichlet boundary condition. Commun. Pure Appl. Anal. 14, 2095-2115 (2015)

11. Zhao, X-Q: Global attractivity in a class of nonmonotone reaction diffusion equations with time delay. Can. Appl. Math. Q. 17, 271-281 (2009)

12. Thieme, HR, Zhao, X-Q: A non-local delayed and diffusive predator-prey model. Nonlinear Anal., Real World Appl. 2, 145-160 (2001)

13. Evans, LC: Partial Differential Equations. Graduate Studies in Mathematics, vol. 19. Am. Math. Soc., Providence (1998)

14. Gurney, WSC, Blythe, SP, Nisbet, RM: Nicholson's blowflies revisited. Nature 287, 17-21 (1980)

15. Cooke, $\mathrm{K}$, van den Driessche, $\mathrm{P}, \mathrm{Zou}, \mathrm{X}$ : Interaction of maturation delay and nonlinear birth in population and epidemic models. J. Math. Biol. 39, 332-352 (1999)

16. Faria, T: Asymptotic stability for delayed logistic type equations. Math. Comput. Model. 43, 433-445 (2006)

17. Györi, I, Trofimchuk, S: Global attractivity in $x^{\prime}(t)=-\delta x(t)+p f(x(t-\tau))$. Dyn. Syst. Appl. 8, $197-210(1999)$

18. Ivanov, AF, Sharkovsky, AN: Oscillations in singularly perturbed delay equations. Dyn. Rep. 1, 164-224 (1992)

19. Krisztin, T: Global dynamics of delay differential equations. Period. Math. Hung. 56, 83-95 (2008)

20. Krisztin, T, Walther, H-O, Wu, J: Shape, Smoothness and Invariant Stratification of an Attracting Set for Delayed Monotone Positive Feedback. Am. Math. Soc., Providence (1999)

21. Liz, E: Four theorems and one conjecture on the global asymptotic stability of delay differential equations. In: The First 60 Years of Nonlinear Analysis of Jean Mawhin, pp. 117-129. World Scientific, Singapore (2004)

22. Liz, E, Rost, G: On the global attractivity of delay differential equations with unimodel feedback. Discrete Contin. Dyn. Syst. 24, 1215-1224 (2009)

23. Röst, $G, W u$, J: Domain-decomposition method for the global dynamics of delay differential equations with unimodal feedback. Proc. R. Soc. Lond. Ser. A 463, 2655-2669 (2007) 
24. Walther, H-O: The 2-dimensional attractor of $x^{\prime}(t)=-x(t)+f(x(t-1))$. Mem. Am. Math. Soc. 113, 554 (1995)

25. Smith, HL: Monotone Dynamical Systems: An Introduction to the Theory of Competitive and Cooperative Systems. Mathematical Surveys and Monographs, vol. 41. Am. Math. Soc., Providence (1995)

26. Wu, J: Theory and Applications of Partial Functional Differential Equations. Appl. Math. Sci., vol. 119. Springer, New York (1996)

27. Martin, $\mathrm{RH}$, Smith, HL: Abstract functional differential equations and reaction-diffusion systems. Trans. Am. Math. Soc. 321, 1-44 (1990)

28. Amann, H: Fixed point equations and nonlinear eigenvalue problems in ordered Banach space. SIAM Rev. 18, 620-709 (1976)

29. Smith, H: An Introduction to Delay Differential Equations with Applications to the Life Sciences. Texts in Applied Mathematics, vol. 57. Springer, New York (2011)

30. Chern, I-L, Mei, M, Yang, X, Zhang, Q: Stability of non-monotone critical traveling waves for reaction-diffusion equations with time-delay. J. Differ. Equ. 259, 1503-1541 (2015)

31. Liu, Y, Yu, Z, Xia, J: Exponential stability of traveling waves for non-monotone delayed reaction-diffusion equations. Electron. J. Differ. Equ. 2016, 86 (2016)

32. So, JW-H, Wu, J, Zou, X: A reaction diffusion model for a single species with age structure - I. Traveling wave fronts on unbounded domains. Proc. R. Soc. Lond. A 457, 1841-1853 (2001)

33. $\mathrm{Yi}, \mathrm{T}, \mathrm{Chen}, \mathrm{Y}, \mathrm{Wu}, \mathrm{J}$ : Unimodal dynamical systems: comparison principles, spreading speeds and travelling waves. J. Differ. Equ. 254, 3538-3572 (2013)

34. Mackey, MC, Glass, L: Oscillation and chaos in physiological control systems. Science 197, $287-289$ (1977)

\section{Submit your manuscript to a SpringerOpen ${ }^{\circ}$} journal and benefit from:

- Convenient online submission

- Rigorous peer review

Open access: articles freely available online

High visibility within the field

- Retaining the copyright to your article

Submit your next manuscript at springeropen.com 